\title{
Intraventricular Hemorrhage in Full Term Newborn: A Rare Phenomenon
}

\author{
Upma Suneja*, Rekha Gadiparthi, Leyden Standish, Benamanahalli Rajegowda and Sergey \\ Prokhorov
}

Pediatrics Department, Lincoln Medical and Mental Health Center, New York, USA

*Corresponding author: Upma Suneja, Pediatrics Department, Lincoln Medical and Mental Health Center, New York, USA, Tel: 17185795016; Email: drupmasuneja@yahoo.com

Received date: Jul 29, 2016; Accepted date: Aug 04, 2016; Published date: Aug 11, 2016

Copyright: ㄷ 2016 Suneja U, et al. This is an open-access article distributed under the terms of the Creative Commons Attribution License, which permits unrestricted use, distribution, and reproduction in any medium, provided the original author and source are credited.

Citation: Suneja U, Gadiparthi R, Standish L, et al. Intraventricular Hemorrhage in Full Term Newborn: A Rare Phenomenon. Arch Med. 2016, $8: 4$

\section{Introduction}

Intraventricular hemorrhage, also known as sub ependymal hemorrhage signifies the bleeding in and around the ventricles. The incidence of IVH has declined from 40 to $50 \%$ in 1970 's to $20-25 \%$ in 2010 , but the absolute numbers still continue to remain significant. The risk of having IVH is inversely related to gestational and birth weight, reason why it is quite uncommon in full term neonates. Here, we present a case of full term neonate born by normal vaginal delivery and presented with seizures on second day of life. He was found to have grade $2 \mathrm{IVH}$ upon imaging.

\section{Case}

A baby boy was born full term, appropriate for age, by normal vaginal delivery to 29 year old G8P0070 mother with history of multiple previous miscarriages and maternal hypothyroidism (well controlled on synthroid). The baby at birth had APGAR of $9 / 9$ at 1 and 5 minutes and physical examination was unremarkable. The baby was admitted to normal newborn nursery for regular nursery care and mother newborn bonding. The baby was feeding well and voiding freely. On DOL \# 2, the baby was noted to have jerky movements of all the four extremities that did not diminish with repositioning or holding. The episode lasted for almost 2 minutes followed by 2 minutes of post ictal phase marked by decreased muscle tone. Bedside finger stick was $54 \mathrm{mg} / \mathrm{dl}$ and the physical examination after the episode was remarkable for good muscle tone and pink colour. The decision was made to transfer the baby from nursery to NICU. On arrival to NICU, the baby had another episode of clonic movements of all four extremities with facial twitching that required loading dose of phenobarbital and lasted approximately 15 minutes. A full work up including $\mathrm{CBC}$, chemistry, blood gas, blood culture, Lumbar puncture for CSF cell count, glucose, protein and culture, serum and CSF HSV cultures, serum ammonia level, serum amino acids, urine organic acids and urine toxicology were done, all of which were unremarkable. The patient was empirically started on ampicillin, gentamicin and acyclovir, which were discontinued later on after the cultures were reported negative. Neurology was consulted who recommended to do Head CT without contrast which was remarkable for small $(0.9 \times 0.5 \mathrm{~cm})$ hemorrhage at the caudothalamic groove on the right consistent with germinal matrix hemorrhage (intraventricular hemorrhage: Figure 1). Also, minimal subarachnoid hemorrhages in several sulci near the vertex. No evidence of midline shift or hydrocephalus.

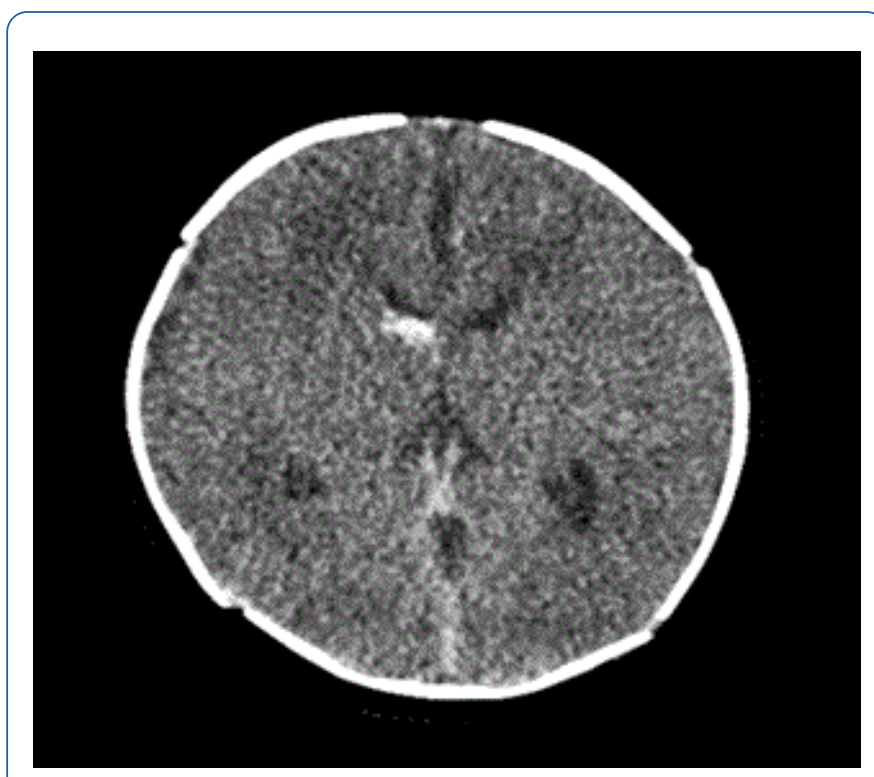

Figure 1 Intraventricular hemorrhage seen as hyperechoic area (arrow).

Neurology recommended to continue close monitoring and to give phenobarbital only in case of recurrent seizure. Also, considering the maternal history of multiple miscarriages, mother was worked up for antiphospholipid syndrome which was negative. Upon further questioning, mother reported positive family history of seizure disorder in maternal uncle and grandmother. The baby was followed as inpatient over a period of next 5 days during which he remained seizure and was discharged home on DOL \# 7 with follow up in neurology clinic. In neurology clinic follow ups, the baby is found to have gross motor delays and bilateral ptosis. The patients being 
followed in neurology clinic regularly and the bilateral ptosis seems to persist without progression.

\section{Discussion}

Intraventricular hemorrhage, mostly seen as a complication in low birth weight and preterm infants, is a rare occurrence in full term neonates. In a large retrospective review of cranial ultra-sonographic findings in healthy full term neonates, $1.9 \%$ neonates were reported to have mild intraventricular hemorrhage. The bleeding usually starts in sub ependymal region and can extend either way to the ventricles or into the parenchyma. The immaturity of the vasculature and the central nervous system regulation are the major factors leading to hemorrhage in the preterm neonates. Apart from prematurity, other prominent causes include rapid volume expansion, perinatal mechanical trauma, perinatal asphyxia and coagulopathies.

In a National Inpatient Sample database analysis, more boys than girls were found to have severe IVH (38\% vs $32.7 \%$, $\mathrm{p}<0.004)[1,2]$. The highest risk for IVH is during the first 3 days of life after which the germinal matrix vessels become more robust [3]. Based on the extent of hemorrhage evident on imaging, IVH is classified from Grade I to grade IV. Most of the patients are asymptomatic, however when symptomatic may present with seizures, hypotension, hypotonic or high pitched cry. Most of the grade I-II hemorrhages resolve spontaneously without any intervention. Grade III-IV may require intervention. Immediate goal of treatment would be to treat the complications including hypotension, acidosis, seizures, and anemia. Long term prognosis depends on grade of IVH with extensive bilateral hemorrhages resulting in persistent motor and cognitive deficits. Prenatal use of betamethasone has shown to provide some protection against IVH. Postnatal use of indomethacin for prevention of IVH is under trial $[4,5]$.

\section{Conclusion}

IVH, a well-known complication in preterm neonates is rarely seen in full term babies. Perinatal trauma may be an important contributing factor in these cases. Since most of the cases are asymptomatic or present with subtle signs and symptoms, a careful newborn examination is essential. Through this presentation, we intend to bring in attention that IVH should be considered as a differential even for a full term neonate presenting with neurological findings.

\section{References}

1. Mohamed MA, Aly H (2010) Male gender is associated with intraventricular hemorrhage. Pediatrics 125: e333-e339.

2. Volpe (2008) Neurology of the newborn.

3. Whitelaw A (2011) Core Concepts: Intraventricular hemorrhage. Neoreviews 12: e94.

4. Dykes FD, Lazzara A, Ahmann P, Blumenstein B, Shwartz J, et al. (1980) Intraventricular hemorrhage: A prospective evaluation of etiopathogenesis. Pediatrics 66: 42.

5. Hsu CL, Lee KL, Jeng MJ, Chang KP, Yang CF, et al. (2012) Cranial ultrasonographic findings in healthy full term neonates: a retrospective review. J Chin Med Assoc 75: 386-395. 